\title{
Semiconductor saturable absorber mirror structures with low saturation fluence
}

\author{
Journal Article \\ Author(s): \\ Spühler, G.J.; Weingarten, K.J.; Grange, R.; Krainer, L.; Haiml, M.; Liverini, V.; Golling, M.; Schön, S.; Keller, Ursula \\ Publication date: \\ 2005-07 \\ Permanent link: \\ https://doi.org/10.3929/ethz-b-000032386 \\ Rights / license: \\ In Copyright - Non-Commercial Use Permitted \\ Originally published in: \\ Applied Physics B 81(1), https://doi.org/10.1007/s00340-005-1879-1
}


G.J. SPÜHLER ${ }^{1}$

K.J. WEINGARTEN ${ }^{2}$

R. GRANGE ${ }^{1}$

L. KRAINER ${ }^{1}$

M. HAIML ${ }^{1}$

V. LIVERINI ${ }^{1}$

M. GOLLING ${ }^{1}$

S. SCHÖN ${ }^{1}$

U. KELLER ${ }^{1, \infty}$

\section{Semiconductor saturable absorber mirror structures with low saturation fluence}

\author{
${ }^{1}$ ETH Zurich, Physics Department, Institute of Quantum Electronics, Wolfgang-Pauli-Strasse 16, \\ 8093 Zürich, Switzerland \\ ${ }^{2}$ Time-Bandwidth Products, GigaTera Product Group, Technoparkstr. 1, 8005 Zürich, Switzerland
}

\section{Received: 27 April 2005}

Published online: 9 June 2005 • Springer-Verlag 2005

ABSTRACT We present two novel semiconductor saturable absorber mirror (SESAM) designs which can exhibit more than ten times lower saturation fluence than classical SESAM devices. Design considerations and characterization data are presented. These devices are particularly suited for passively mode-locked lasers with ultra-high repetition rates.

PACS 42.60.Fc; 78.67.Pt; 42.70.Nq

1

\section{Introduction and motivation}

Semiconductor saturable absorber mirrors (SESAM devices) [1, 2] are well established for passive mode locking or Q-switching of many kinds of solid-state lasers [3-5]. Since both linear and nonlinear optical properties of these devices can be engineered over a wide range, the device performance can be readily optimized for a wide variety of laser designs and operating regimes. The main device parameters such as operation wavelength $\lambda$, modulation depth $\Delta R$, saturation fluence $F_{\text {sat }}$, and absorber recovery time $\tau_{\mathrm{A}}$ can be custom designed in a wide range for stable continuous-wave (cw) mode locking [6], pure Q-switching [7], or a combination of both [3].

One important limit of a SESAM device is its saturation fluence, which has typical values in the range of several tens to hundreds of $\mu \mathrm{J} / \mathrm{cm}^{2}$. Lower saturation fluence is particularly relevant for fundamentally mode-locked solid-state lasers with an ultra-high pulse-repetition rate (i.e. $\gg 1 \mathrm{GHz}$ ) [8-10]. It becomes harder to saturate the SESAM device in such a laser, as the intracavity pulse energy becomes increasingly lower, requiring laser mode sizes on the SESAM device on the order of only a few microns (i.e. close to the diffraction limit). Additionally, the very short cavity length required for such high repetition rates does not leave much room for a cavity design with arbitrarily small mode sizes on the SESAM device. Furthermore, the threshold for continuous-wave mode locking without Q-switching instabilities (QML threshold) is decreased with a low saturation fluence [6]. It is also worth noting that passively mode-locked vertical external cavity

Fax: +41-44-633-10-59, E-mail: keller@phys.ethz.ch semiconductor lasers (VECSELs) can also benefit from a SESAM device with low saturation fluence [11, 12].

Novel absorber materials with increased absorption cross sections are one alternative to reduce the saturation fluence. Quantum dots are promising candidates for this [12, 13], and in the $1.3-1.5-\mu \mathrm{m}$ wavelength regime GaInNAs absorbers can show decreased saturation fluence [14-16]. For this paper, we investigate specific structural changes that can be done to the SESAM multilayer design in order to obtain a device with lower saturation fluence, and describe two novel designs with significantly reduced saturation fluence. Of course, improved absorber materials can also be combined with improved design structures.

The concept of a resonant Fabry-Perot microstructure is well known [17] and forms the basis for many optical switching and bistable devices and active devices (such as VCSELs, etc.). These structures typically consist of a bottom distributed Bragg reflector (DBR), an active region corresponding to the Fabry-Perot cavity, and a top DBR. For example, an optical switch would have a nonlinear material (e.g. a quantum-well superlattice), with the cavity length adjusted for resonance, i.e. the intracavity intensity would be much higher than the incident intensity. Such devices are well suited for high-contrast optical switching. The first SESAM device for solid-state lasers, the anti-resonant Fabry-Perot saturable absorber, consisted of a Fabry-Perot cavity filled with a saturable absorber, and with its thickness adjusted for anti-resonance, i.e. the intensity in the cavity was substantially lower than the incident intensity [1]. Later, we recognized that the top mirror element was not necessary, and that the absorber could be integrated in the lower Bragg mirror [18, 19] or an appropriate spacer layer [2, 19]. Earlier, a nonlinear Bragg reflector was introduced by Garmire's group but had too much loss modulation to be suitable for passively mode locking solid-state lasers at the time [20]. A close-to-resonant Fabry-Perot saturable absorber structure, referred to as the D-SAM (dispersive saturable absorber mirror) was used to optimize both negative group-delay dispersion (GDD) and the nonlinear modulation to construct more compact femtosecond sources [21].

Here, we discuss two new structures which allow for tailoring of the device's saturation fluence and GDD as well as the modulation depth. We choose our design wavelength to 


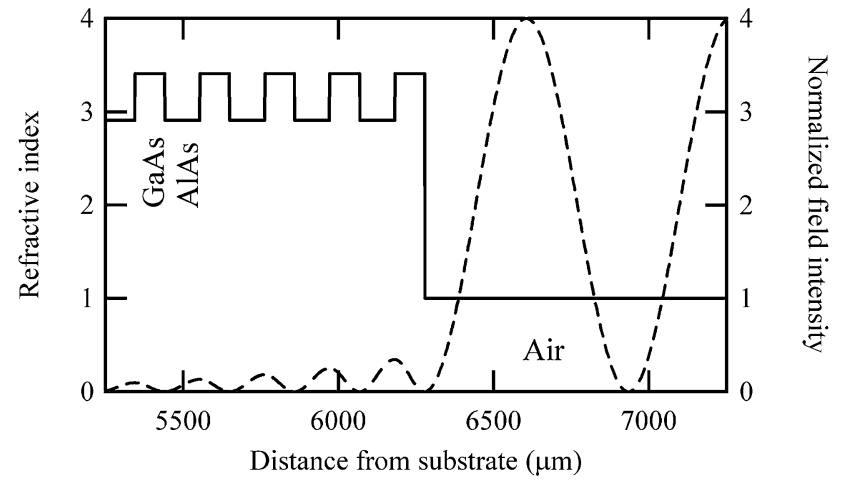

FIGURE 1 Refractive-index structure (solid) and calculated standing wave intensity pattern for $\lambda=1314 \mathrm{~nm}$ (dashed) of a 'standard' DBR close to the surface of the device. Definitions of the terms and the structures of the devices can be found in the text

be $1314 \mathrm{~nm}$, motivated by the work in the second telecom window with Nd:YLF lasers. However, the concepts and arguments hold for arbitrary wavelengths. For clarity, we begin our explanation with an illustration of the field intensity in a 'standard' DBR.

\section{$2 \quad$ DBR structure and field intensity enhancement factor $\xi$}

Figure 1 shows the layer structure and field intensity near the top of the structure (this and all further field structures are calculated using a transfer-matrix formalism). In this case, our DBR consists of 30 pairs of alternating quarter-wave layers of AlAs as low-index material ( $n=$ 2.91 at $1314 \mathrm{~nm}$; quarter-wave layer thickness $112.9 \mathrm{~nm}$ ) and GaAs as high-index material ( $n=3.41$ at $1314 \mathrm{~nm}$; quarterwave layer thickness $96.3 \mathrm{~nm}$ ). Incoming light with intensity normalized to 1 creates a standing wave pattern in this structure. In this definition, the peak of the field intensity (square of the envelope of the electric field) of the resulting standing wave pattern is 4 outside the device, assuming a mirror that has a reflectivity of $100 \%$ and negligible loss. We define the enhancement factor $\xi$ as the maximum field intensity in the structure relative to 1 (i.e. relative to the incoming field intensity).

In the DBR example, no absorber layer is shown. In order to introduce saturable absorption (nonlinear reflectivity), an absorber layer would typically be positioned at or very near the maximum standing wave peak inside the structure (resulting in lowest saturation fluence for the device). When a thin absorber layer is added, we can make the assumption that the absorption plays a negligible role in the field distribution, as long as the total absorption is small, i.e. on the order of a few percent. Similarly, any change in index of the thin absorber layer has negligible impact on the field distribution, since reflections from the individual surfaces nearly cancel out due to destructive interference. The expected modulation depth of the device with absorber layer is then directly proportional to $\xi$, and the saturation fluence inversely proportional to $\xi[22]$, for a given absorber layer thickness and material.

Considering the field intensity in this 'standard' DBR, we see in Fig. 2 that the $\xi$ factor is approximately 0.34 , and

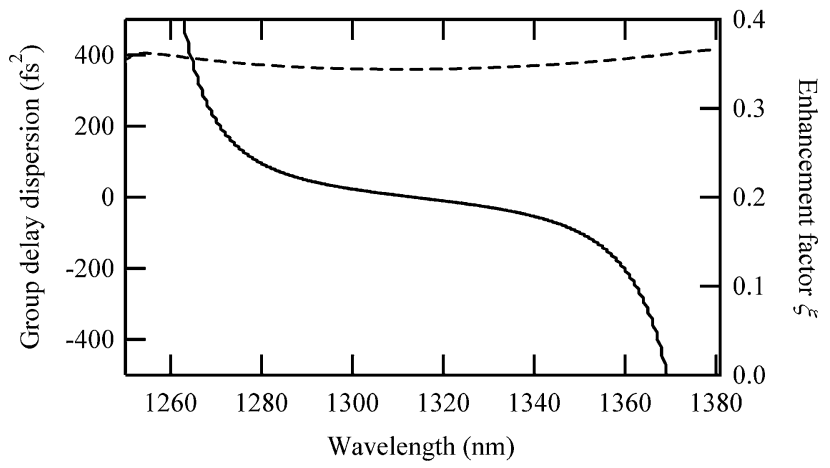

FIGURE 2 Calculated group-delay dispersion (solid) and enhancement factor $\xi$ (dashed) as a function of operation wavelength for 'standard' DBR

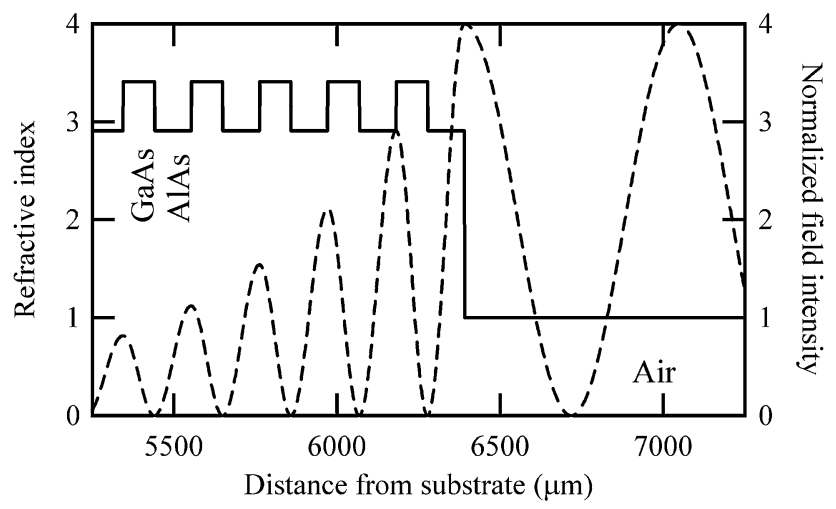

FIGURE 3 Refractive-index structure (solid) and calculated standing wave intensity pattern for $\lambda=1314 \mathrm{~nm}$ (dashed) of a 'resonant-like' DBR close to the surface of the device

the GDD and $\xi$ are nearly flat over the center-wavelength range. This device does not exhibit any resonant-like behavior in either the field intensity or the GDD. Note that we can make the following comments on this DBR structure: the last quarter-wave layer is a high-index layer, and accordingly the field intensity at the air interface ends on or very near a node (i.e. a field null).

Now consider a DBR structure with an additional quarterwave layer of low-index material placed on top of the structure (Fig. 3). This results in several key feature changes to the field intensity in the structure and the device's GDD behavior (Fig. 4). First, the $\xi$ factor now equals 4 , i.e. it is enhanced with respect to the 'standard' DBR by a factor of more than 11. Secondly, the device exhibits some peaking of the group delay, resulting in significant GDD in the device away from its design-operating point. This peaking of the group delay is 'resonant-like' behavior. However, the field intensity in the device is never larger than the external intensity. Also note that the air-device interface characteristically ends on or near a peak (anti-node) of the field intensity.

Note that we have chosen to add a quarter-wave layer of the low-index material of the DBR. However, we are free to add a quarter-wave layer of any index material, and still achieve similar results, i.e. an increase of the $\xi$ factor to nearly 4, and an enhanced GDD response. A higher-index layer results in more peak GDD, and a lower-index layer results in less peak GDD. 


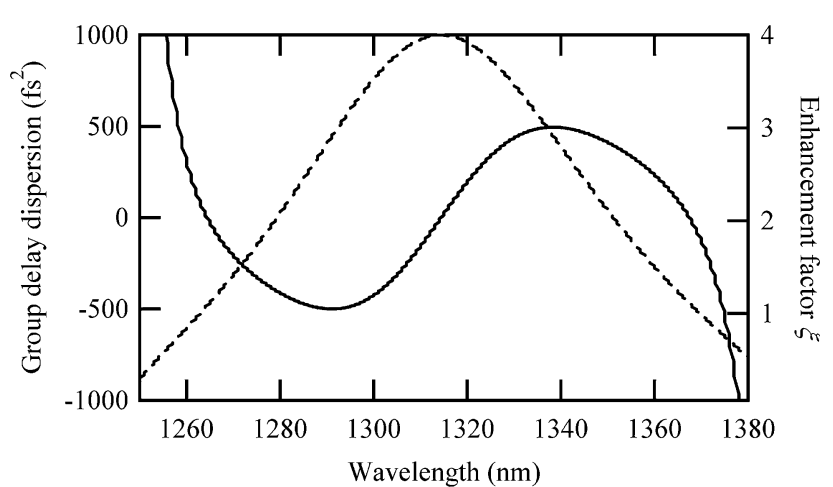

FIGURE 4 Calculated group-delay dispersion (solid) and enhancement factor $\xi$ (dashed) as a function of operation wavelength for 'resonant-like' DBR

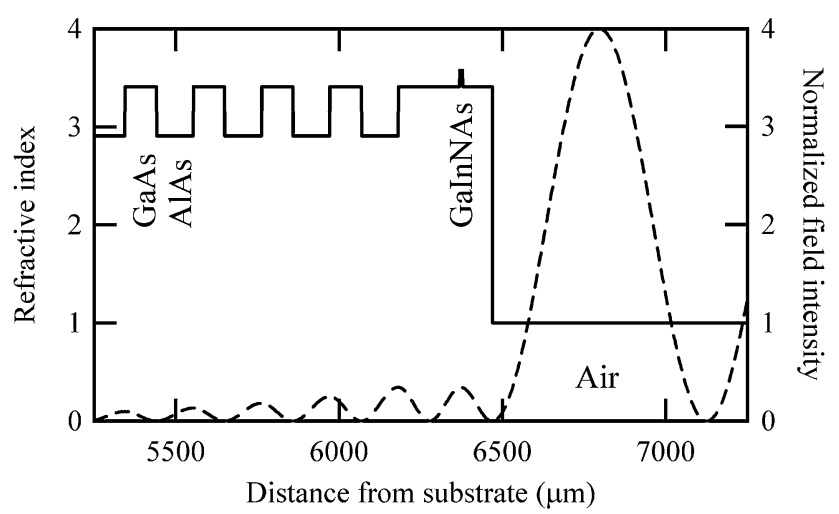

FIGURE 5 Refractive-index structure (solid) and calculated standing wave intensity pattern for $\lambda=1314 \mathrm{~nm}$ (dashed) of a 'classical' SESAM device structure close to the surface of the device

\section{'Classical' SESAM structure}

Now we consider a 'classical' SESAM device structure (Fig. 5). A possible design for a wavelength of $1314 \mathrm{~nm}$ consists of a 30-pair AlAs/GaAs standard DBR grown on a GaAs substrate, and ending on a GaAs quarterwave layer, and then an approximately half-wave layer consisting of the following: 91-nm GaAs, 10-nm GaInNAs absorber layer, and another $91 \mathrm{~nm}$ of GaAs. This SESAM device exhibits a low enhancement factor of $\xi=0.34$ and it shows flat, non-resonant-like behavior (Fig. 6). Note that the field intensity at the device-air interface ends on a node.

In contrast to the classical SESAM device, a high-finesse, highly resonant Fabry-Perot device could easily yield $\xi$ values higher or much higher than unity [17] and therefore yield much lower saturation fluences. Such devices have the tradeoff that their optical properties ( $F_{\text {sat }}, \Delta R$, losses, and GDD) become increasingly narrowband as $\xi$ increases, and are increasingly sensitive to ambient changes and growth tolerances due to the sharp resonance. For example, the GDD would no longer show a flat response over the center of the mirror reflectivity, but would show a peaked, resonant response. However, for devices which require only a limited operation bandwidth of a few nanometers, some enhancement of the $\xi$ factor can be useful, limited by the above trade-offs. Here, we describe two approaches to achieve an enhancement of the intradevice fields, without introducing a top mirror element. We introduce the terms 'low-field-enhancement

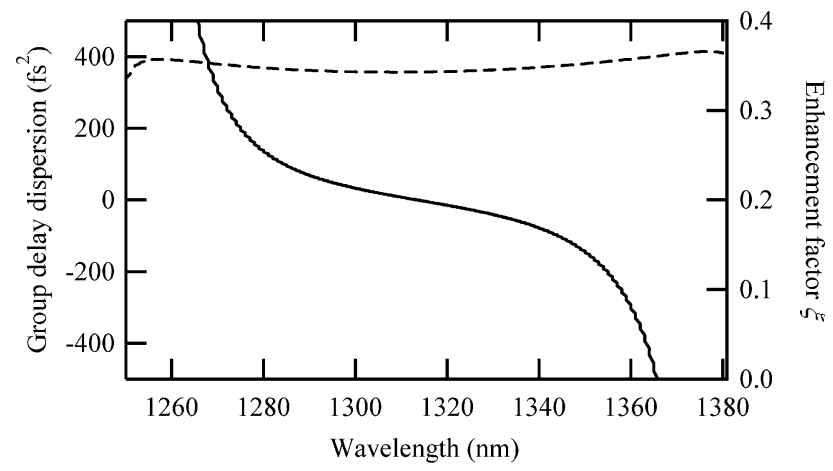

FIGURE 6 Calculated group-delay dispersion (solid) and enhancement factor $\xi$ (dashed) as a function of operation wavelength for a 'classical' SESAM device

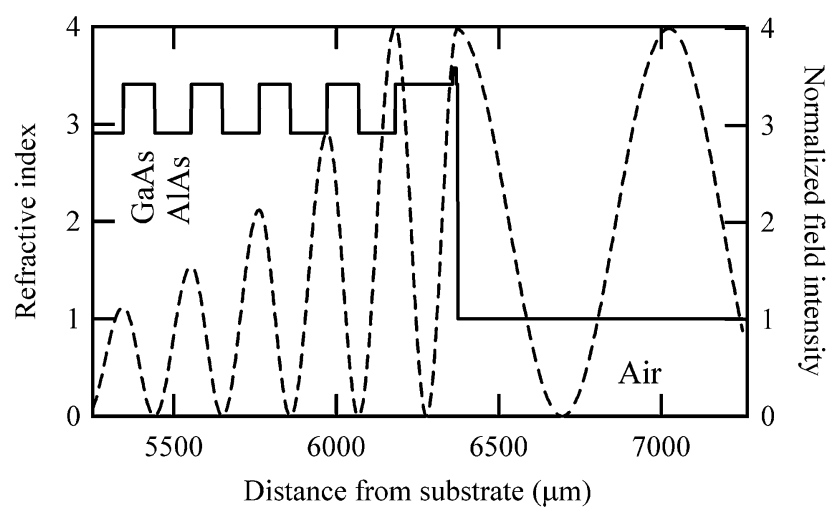

FIGURE 7 Refractive-index structure (solid) and calculated standing wave intensity pattern for $\lambda=1314 \mathrm{~nm}$ (dashed) of a LOFERS structure close to the surface of the device

resonant-like SESAM device' (LOFERS) [23] and 'enhanced SESAM device' (E-SESAM) [24].

\section{4}

\section{LOFERS structure}

Consider the 'low-field-enhancement resonantlike SESAM device' (LOFERS) [23] shown in Fig. 7. We start with a 30-pair DBR but extend the top quarter-wave layer of GaAs by approximately an additional quarter-wave layer. This is similar to the 'resonant-like' DBR described, but the top layer is the high-index material (GaAs). The absorber is placed near the surface, and a very thin GaAs cap layer is added to protect the absorber layer: e.g. 80.8nm GaAs spacer layer, 10-nm GaInNAs absorber layer, and 5-nm GaAs cap. Figure 7 shows the refractive index as well as the calculated field intensity near the surface of the structure. Figure 8 shows the $\xi$ factor and GDD as a function of wavelength. The field in the absorber is nearly equal to the outside field: $\xi=3.90$. Relative to the classical low-finesse SESAM device $(\xi=0.34)$, this is a field enhancement by a factor of approximately 11 , implying that the saturation fluence should be 11 times lower and the modulation depth 11 times higher. Also note that the peak GDD value is approximately $1100 \mathrm{fs}^{2}$, a few tens of nanometers away from the designed operation wavelength. The LOFERS design clearly uses the field enhancement in the saturable absorber layer, which is different to the D-SAM design [21], mentioned above, where the absorber was close to a node of the standing wave field intensity. 


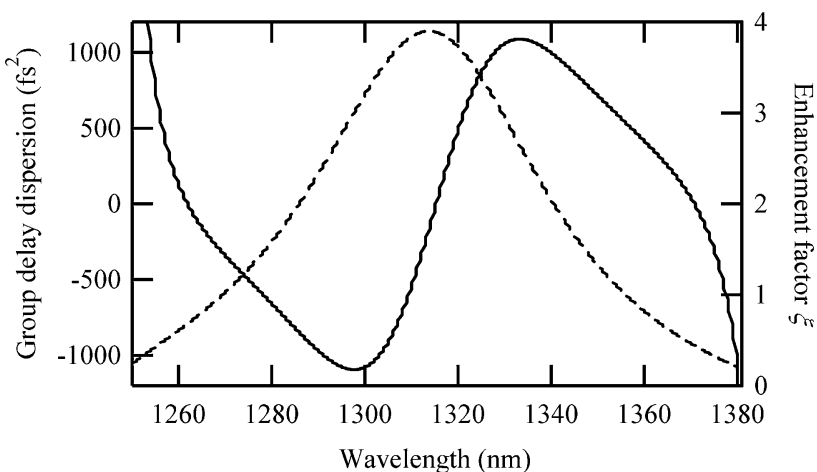

FIGURE 8 Calculated group-delay dispersion (solid) and enhancement factor $\xi$ (dashed) as a function of operation wavelength for a LOFERS structure

Any non-saturable losses within the structure (as opposed to surface scattering) will also scale with $\xi$ as well. This can lead to a reduced efficiency of the laser or even to absorber damage. However, as most of the losses are typically introduced by defects induced by the strain (or special growth conditions, e.g. low-temperature growth $[25,26])$ in the absorber layer, a reduced absorber thickness in the LOFERS structure can help to reduce both $\Delta R$ and the non-saturable losses (with minimal impact on $F_{\text {sat }}$ ). In this way, practical low modulation depths of a few tenths of a percent can be obtained (similar to those of classical SESAM devices) with a significantly reduced $F_{\text {sat }}$ (compared to the classical SESAM device). Furthermore, the QML threshold is reduced, as it depends on $\sqrt{F_{\text {sat }} \times \Delta R}[6,8]$. Due to the increased field in the layers, the transmission losses also increase. To maintain the same reflectivity as a classical SESAM device, an increased number of Bragg pairs may be desirable (i.e. 30 pairs at least).

Referring to Fig. 8, one sees that the stronger dependence of $\xi$ and GDD on $\lambda$ indicates that the operation bandwidth and consequently the growth tolerances of a LOFERS are reduced. Also, the GDD values of up to over $1000 \mathrm{fs}^{2}$ can lead to non-transform-limited pulses. In spite of these limitations, the LOFERS can be a very useful device for a laser whose emission (tuning range or bandwidth) is limited to a few nanometers, as within this range the variation of the main absorber parameters is acceptably low.

\section{E-SESAM structure}

The 'enhanced SESAM device' (E-SESAM) [24] is an intermediate solution between the LOFERS and the classical SESAM device. In this case, a LOFERS-type structure is completed with a single low-index (i.e. lower than the low-index DBR material) dielectric quarter-wave layer. In our example here, we deposit a quarter-wave layer of $\mathrm{SiO}_{2}$ at the surface (Fig. 9). This yields a relatively high enhancement factor of $\xi=1.77$, slightly less than half of the LOFERS, but still more than five times enhanced compared to the classical SESAM device. The GDD of this structure is now nearly wavelength independent and close to zero in the regime of interest (Fig. 10). The field intensity has a node on or near the surface of the device. Also, the wavelength dependence of $\xi$ is

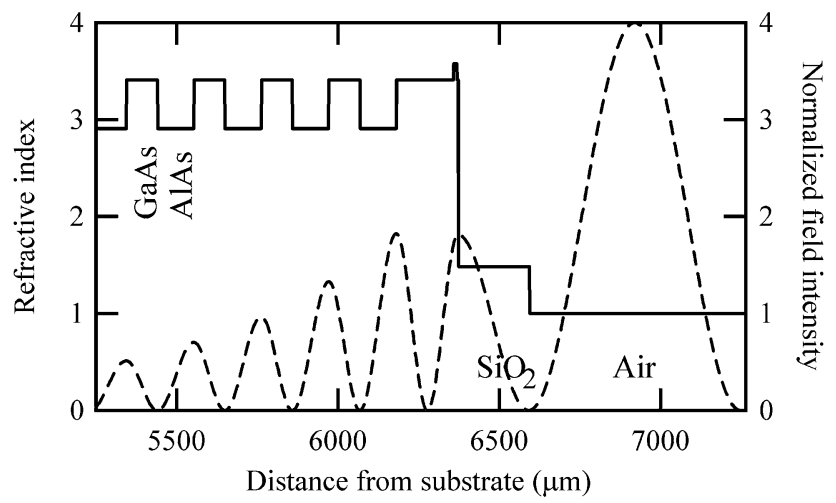

FIGURE 9 Refractive-index structure ( solid) and calculated standing wave intensity pattern for $\lambda=1314 \mathrm{~nm}$ (dashed) of an E-SESAM structure close to the surface of the device

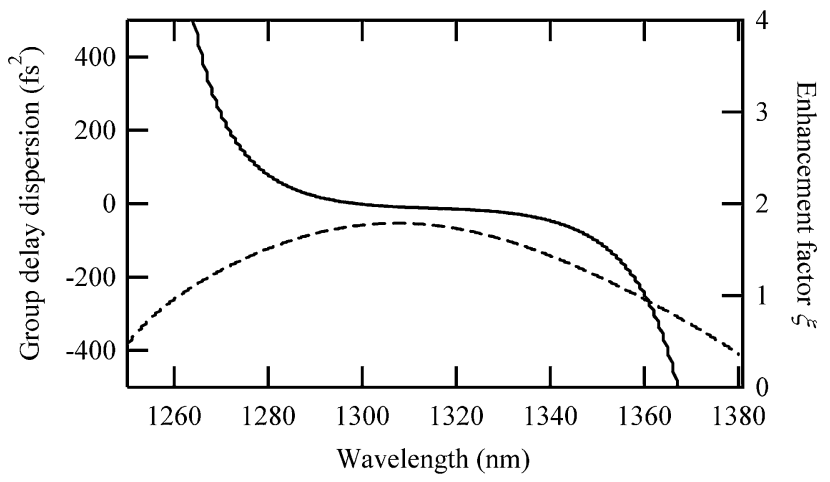

FIGURE 10 Calculated group-delay dispersion (solid) and enhancement factor $\xi$ (dashed) as a function of operation wavelength for an E-SESAM structure

strongly reduced. Therefore, this device can be a better choice for tunable lasers or lasers with larger operating bandwidth. Additionally, the growth tolerances are relaxed compared to the LOFERS, at the expense of a post-processing step. However, the enhancement layer can serve as passivation, and it can be used to compensate for possible growth errors.

\section{LOFERS vs. E-SESAM and extended concepts}

The LOFERS and E-SESAM concepts can be further extended for a broader range of device parameters. As a general design guideline, we can make the following formulations. First, one can select and design an appropriate classical SESAM device, which has a $\xi$ factor of approximately 0.34 . If this does not have the desired modulation depth or saturation fluence, we can add an approximate quarter-wave layer of any optical material to obtain a LOFERS structure. If we choose to add the high-index material (GaAs), we can add this to the structure during its epitaxial growth, without removing it from the growth chamber, resulting in a field enhancement of up to 11 times the classical SESAM device. Note that we can either add an approximate quarter wave of GaAs to the classical SESAM device (corresponding to a final layer of approximately three-quarter-wave thickness of GaAs) or similarly we can 'shorten' the last GaAs layer of the classical SESAM device by approximately a quarter wave. In either case, the absorber is positioned near the peak of the field, either near the air interface of the last layer or at the next 
peak at approximately a half wave inward from the GaAs-air interface.

As an alternative to this 'GaAs LOFERS', the LOFERS structure can be fabricated after epitaxial growth of the classical SESAM structure, by deposition of any appropriate dielectric material layer of approximately quarter-wave thickness. Although the field intensity at the start of this layer is 4 , because the absorber is still embedded in an underlying GaAs layer, these types of LOFERS have a reduced $\xi$ factor and a reduced peak GDD value. The lower the index of refraction of the dielectric, the lower the $\xi$ factor. In all cases, the standing wave field intensity in the LOFERS is near a peak at the device-air interface.

For applications which require generally flatter GDD response and do not require $\xi$ factors as high as from a LOFERS, one can choose an E-SESAM structure. The E-SESAM would nominally consist of the LOFERS described above (i.e. DBR plus quarter-wave or three-quarterwave high-index layer, with absorber near a field peak) plus an approximate quarter-wave layer of a material with typically lower refractive index, which we call an enhancement layer. Note that if this enhancement layer was GaAs, we would be back to a structure nearly identical to the classical SESAM device, with a $\xi$ factor of about 0.34 and a flat GDD close to zero. Choosing the refractive index of the enhancement layer to be lower than the refractive index of both DBR materials, this $\xi$ factor can be adjusted to any value from 0.34 to greater than 2 . The E-SESAM generally shows flatter GDD response and relaxed growth tolerances compared to the LOFERS structure.

Even more generally (and requiring typically full matrix formalism calculations), the layer thicknesses can be adjusted so that one can obtain structures with $\xi, F_{\text {sat }}$, and GDD values in an even wider range (i.e. the layer thickness must not be restricted to quarter-wave multiples). The appropriate modulation depth can then be achieved by adjusting the thickness of the absorber layer within the physical limits. Further, it is possible to decrease the modulation depth by moving the absorber layer away from the peak of the field intensity. However, this is accompanied by an increase of saturation fluence. Note that although we have described typical structures based on GaAs and AlAs, other material combinations would certainly be possible to achieve similar structures and similar performance values.

\section{7}

\section{Experimental confirmation of the structure concepts}

We have fabricated a SESAM device and a LOFERS, similar to the structures described above. The classical SESAM device consists of a 79-nm spacer layer, a 10-nm GaInNAs absorber layer, and a 122-nm GaAs cap layer, grown by molecular beam epitaxy on top of a 30-pair AlAs/GaAs DBR [15]. The calculated $\xi$ value is 0.29 . The LOFERS structure is grown on the same DBR and consists of identical 79-nm spacer layer and 10-nm GaInNAs absorber layer, and a 10-nm GaAs cap layer [15]. We calculate $\xi=2.75$ for this LOFERS structure. Figure 11 shows the measurement of nonlinear reflectivity as a function of fluence incident on the samples [27], fitted

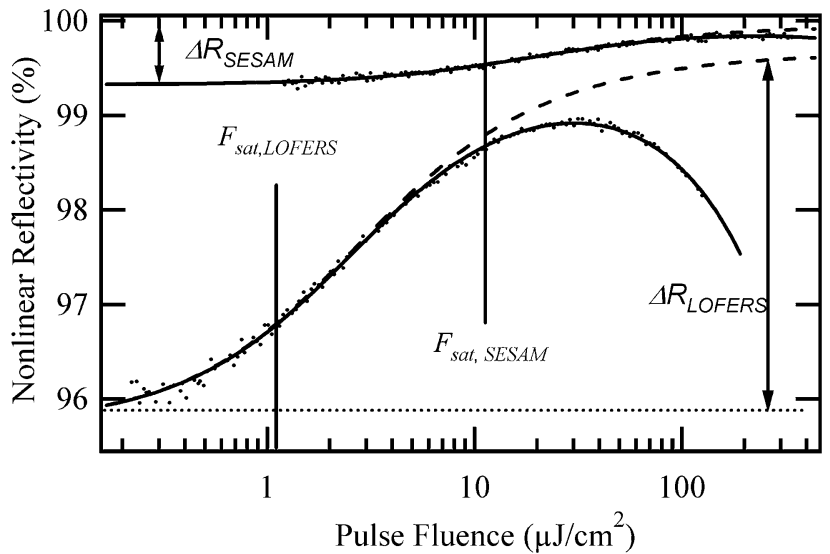

FIGURE 11 Nonlinear reflectivity measurements (dots) of the GaInNAs SESAM device (upper curves) and LOFERS lower curves) with least-square fits using an absorber model including a second-order induced absorption (solid line) or without induced absorption (dashed line) [28]. Measured with 280 -fs pulses at a center wavelength of $1314 \mathrm{~nm}$ and a pulse-repetition rate of $80 \mathrm{MHz}$

with least-square fits using an absorber model including a second-order induced absorption or without induced absorption [28]. For the classical SESAM device, we measure $F_{\text {sat }}=11.2 \mu \mathrm{J} / \mathrm{cm}^{2}, \Delta R=0.6 \%$, and non-saturable losses of $0.04 \%$. For the LOFERS structure, we measure $F_{\text {sat }}=1.1 \mu \mathrm{J} / \mathrm{cm}^{2}, \Delta R=3.9 \%$, and non-saturable losses of $0.3 \%$. The LOFERS exhibits a $\approx 10$ times smaller saturation fluence, $\mathrm{a} \approx 7$ times higher modulation depth, and $\mathrm{a} \approx 8$ times higher loss than the SESAM device. These factors are in reasonable experimental agreement with the ratio of the calculated enhancement factors, which is 9.5. In Ref. [29] we presented a passively mode-locked $1.3-\mu \mathrm{m} \mathrm{Nd}: \mathrm{YVO}_{4}$ laser with a pulse-repetition rate of $10 \mathrm{GHz}$, making use of the low saturation fluence of a GaInNAs E-SESAM as described in this paper. This demonstrates the suitability of this novel approach to reduce the saturation fluence of semiconductor saturable absorber mirrors even further beyond the range accessible with pure material engineering.

\section{8}

\section{Conclusion}

In conclusion, we have presented two novel concepts for semiconductor saturable absorber structures with decreased saturation fluence: the low-field-enhanced resonant-like SESAM device (LOFERS), which gives up to 11 times lower saturation fluence than the classical SESAM device, but at the price of reduced operation bandwidth and tightened growth tolerance, and the enhanced SESAM device (E-SESAM), which combines broadband operation, relaxed growth tolerances, and reduced saturation fluence. We have shown experimental data confirming the calculated values for the classical SESAM device and the LOFERS. These novel absorber structures are demonstrating an important impact in the field of passively mode-locked lasers with high pulserepetition rate.

ACKNOWLEDGEMENTS This work was supported by the Hasler Stiftung, the Quantum Photonics National Center of Competence in Research (QP-NCCR), and the KTI/CTI Swiss Innovation Promotion Agency. 


\section{REFERENCES}

1 U. Keller, D.A.B. Miller, G.D. Boyd, T.H. Chiu, J.F. Ferguson, M.T. Asom, Opt. Lett. 17, 505 (1992)

2 U. Keller, K.J. Weingarten, F.X. Kärtner, D. Kopf, B. Braun, I.D. Jung, R. Fluck, C. Hönninger, N. Matuschek, J. Aus der Au, IEEE J. Sel. Top. Quantum Electron. 2, 435 (1996)

3 U. Keller, Nonlinear Optics in Semiconductors, vol. 59, ed. by E. Garmire, A. Kost (Academic, Boston, MA, 1999), p. 211

4 U. Keller, Prog. Opt. 46, 1 (2004)

5 U. Keller, Nature 424, 831 (2003)

6 C. Hönninger, R. Paschotta, F. Morier-Genoud, M. Moser, U. Keller, J. Opt. Soc. Am. B 16, 46 (1999)

7 G.J. Spühler, R. Paschotta, R. Fluck, B. Braun, M. Moser, G. Zhang, E. Gini, U. Keller, J. Opt. Soc. Am. B 16, 376 (1999)

8 L. Krainer, R. Paschotta, S. Lecomte, M. Moser, K.J. Weingarten, U. Keller, IEEE J. Quantum Electron. 38, 1331 (2002)

9 S.C. Zeller, L. Krainer, G.J. Spühler, R. Paschotta, M. Golling, D. Ebling, K.J. Weingarten, U. Keller, Electron. Lett. 40, 875 (2004)

10 R. Paschotta, L. Krainer, S. Lecomte, G.J. Spühler, S.C. Zeller, A. Aschwanden, D. Lorenser, H.J. Unold, K.J. Weingarten, U. Keller, New J. Phys. 6, 174 (2004)

11 R. Paschotta, R. Häring, U. Keller, A. Garnache, S. Hoogland, A.C. Tropper, Appl. Phys. B 75, 445 (2002)

12 D. Lorenser, H.J. Unold, D.J.H.C. Maas, A. Aschwanden, R. Grange, R. Paschotta, D. Ebling, E. Gini, U. Keller, Appl. Phys. B 79, 927 (2004)

13 S.W. Osborne, P. Blood, P.M. Smowton, Y.C. Xin, A. Stintz, D. Huffaker, L.F. Lester, J. Phys.: Condens. Matter 16, S3749 (2004)

14 M. Kondow, T. Kitatani, S. Nakatsuka, M.C. Larson, K. Nakahara, Y. Yazawa, M. Okai, K. Uomi, IEEE J. Sel. Top. Quantum Electron. 3, 719 (1997)
15 V. Liverini, S. Schön, R. Grange, M. Haiml, S.C. Zeller, U. Keller, Appl. Phys. Lett. 84, 4002 (2004)

16 A. Rutz, R. Grange, V. Liverini, M. Haiml, S. Schön, U. Keller, Electron. Lett. 41, 321 (2005)

17 P.L. Gourley, T.J. Drummond, Appl. Phys. Lett. 49, 489 (1986)

18 L.R. Brovelli, I.D. Jung, D. Kopf, M. Kamp, M. Moser, F.X. Kärtner, U. Keller, Electron. Lett. 31, 287 (1995)

19 I.D. Jung, L.R. Brovelli, M. Kamp, U. Keller, M. Moser, Opt. Lett. 20, $1559(1995)$

20 B.G. Kim, E. Garmire, S.G. Hummel, P.D. Dapkus, Appl. Phys. Lett. 54, 1095 (1989)

21 D. Kopf, G. Zhang, R. Fluck, M. Moser, U. Keller, Opt. Lett. 21, 486 (1996)

22 L.R. Brovelli, M. Lanker, U. Keller, K.W. Goossen, J.A. Walker, J.E. Cunningham, Electron. Lett. 31, 381 (1995)

23 K.J. Weingarten, G.J. Spühler, U. Keller, L. Krainer, GigaTera AG, U.S. Patent 6,538,298 B1 (2003)

24 K.J. Weingarten, G.J. Spühler, U. Keller, S. Thomas, GigaTera AG, U.S. Patent 6,826,219 B2 (2003)

25 M. Haiml, U. Siegner, F. Morier-Genoud, U. Keller, M. Luysberg, R.C. Lutz, P. Specht, E.R. Weber, Appl. Phys. Lett. 74, 3134 (1999)

26 M. Haiml, U. Siegner, F. Morier-Genoud, U. Keller, M. Luysberg, P. Specht, E.R. Weber, Appl. Phys. Lett. 74, 1269 (1999)

27 M. Haiml, R. Grange, U. Keller, Appl. Phys. B 79, 331 (2004)

28 R. Grange, M. Haiml, R. Paschotta, G.J. Spühler, L. Krainer, M. Golling, O. Ostinelli, U. Keller, Appl. Phys. B 80, 151 (2005)

29 G.J. Spühler, L. Krainer, V. Liverini, S. Schön, R. Grange, M. Haiml, A. Schlatter, S. Pawlik, B. Schmidt, U. Keller, Photon. Technol. Lett. Vol. 17, No. 6, p. 1319-1321, (2005) 